(CASE REPORT)

\title{
Severe pneumoperitoneum in a newborn without any established cause in a tertiary health institution in a low resource setting: A case report
}

\author{
Danfulani Mohammed *, Haruna Gele Ibrahim, Shamsuddeen Aliyu and Sule Muhammad Baba \\ Department of Radiology, Faculty of Clinical Sciences, College of Health Sciences, Usmanu Danfodiyo University Sokoto, \\ Nigeria.
}

Publication history: Received on 03 December 2020; revised on 11 December 2020; accepted on 13 December 2020

Article DOI: https://doi.org/10.30574/wjarr.2020.8.3.0481

\begin{abstract}
Pneumoperitoneum is the presence of air within the peritoneal cavity. Pneumoperitoneum is said to occur more in neonates than in infants and most cases are idiopathic. However it may be caused by necrotizing enterocolitis (NEC), gastrointestinal tract perforation, iatrogenic causes such as mechanical ventilation and intrathoracic pathology (pneumothorax, pneumomediastinum). Plain abdominal radiograph has a good diagnostic value in evaluating patients with suspected pneumoperitoneum. Pneumoperitoneum can also be diagnosed using computed tomography (CT). A case of neonatal pneumoperitoneum without any established cause is reported due to its rarity. The approach to its management and radiological signs of pneumoperitoneum are also presented.
\end{abstract}

Keywords: Pneumoperitoneum; Peritoneal cavity; Neonates; Case Report; Computed Tomography

\section{Introduction}

Pneumoperitoneum is the presence of air within the peritoneal cavity. It has an incidence of $16.5 \%$ of neonatal admissions. In neonates, about $10 \%$ of the pneumoperitoneum demonstrated radiologically occur without hollow viscus perforation [1]. Pneumoperitoneum is said to occur more in neonates than in infants and most cases are idiopathic [2]. However it may be caused by necrotizing enterocolitis (NEC), gastrointestinal tract perforation, iatrogenic causes such as mechanical ventilation and intrathoracic pathology (pneumothorax, pneumomediastinum) [3].

Plain abdominal radiograph has a good diagnostic value in evaluating patients with suspected pneumoperitoneum. Pneumoperitoneum can also be diagnosed using computed tomography (CT).

A case of neonatal pneumoperitoneum without any established cause is reported due to its rarity. The approach to its management and radiological signs of pneumoperitoneum are also presented.

\section{Case report}

Baby S. Y. is a one day old male neonate born at 38 weeks gestational age through unsupervised spontaneous vaginal delivery at home. He presented to the special care baby unit of Usmanu Danfodiyo University Teaching Hospital Sokoto 8 hours after delivery with history of gradual and progressive onset of abdominal swelling and difficulty in breathing since birth. No history of vomiting. He had passed meconium before presentation. He is the third child of the mother. No previous history of similar symptoms in the family. The mother received adequate antenatal care which was uneventful. She is not a known diabetic, hypertensive or sickle cell disease patient.

\footnotetext{
${ }^{*}$ Corresponding author: Danfulani Mohammed

Department of Radiology, Faculty of Clinical Sciences, College of Health Sciences, Usmanu Danfodiyo University Sokoto, Nigeria. 
Physical examination showed a male neonate in obvious respiratory distress. The abdomen was grossly distended and soft. He was not pale and not jaundiced. The blood sugar level, electrolytes and packed cell volume were normal. A clinical assessment of grossly distended abdomen? cause to rule out intestinal obstruction was made and he was referred to Radiology department for abdominal radiography.

The plain abdominal radiograph showed large pockets of free peritoneal air under the diaphragms bilaterally (fig.1). The peritoneal air outlined both walls of bowel in keeping with "Rigler's sign" (fig.2). No pneumatosis intestinalis was demonstrated. No evidence of pneumothorax was seen. Abdominal ultrasonography showed excessive intraabdominal gas. The diaphragmatic outline was normal. A diagnosis of pneumoperitoneum probably due to bowel perforation was made.

Percutaneous tapping of the pneumoperitoneum was done to relieve respiratory distress and he later had exploratory laparotomy. At surgery, there was no evidence of gastrointestinal perforation and no pneumatosis intestinalis or congenital anomaly was seen. There was no abdominal distention in the post-operative period. The baby was discharged one week later.

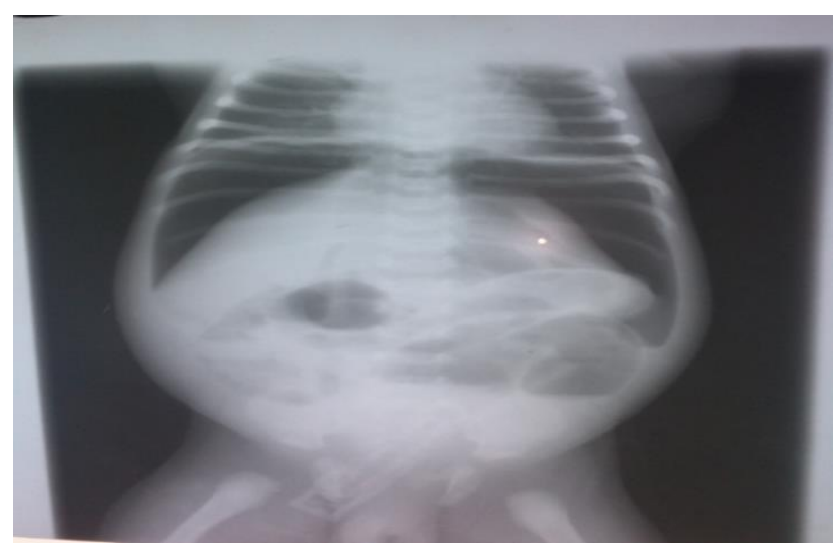

Figure 1 Abdominal radiograph of the abdomen (erect view) showing large volume of air beneath the diaphragm (straight arrows) and Rigler's sign (curved arrow).

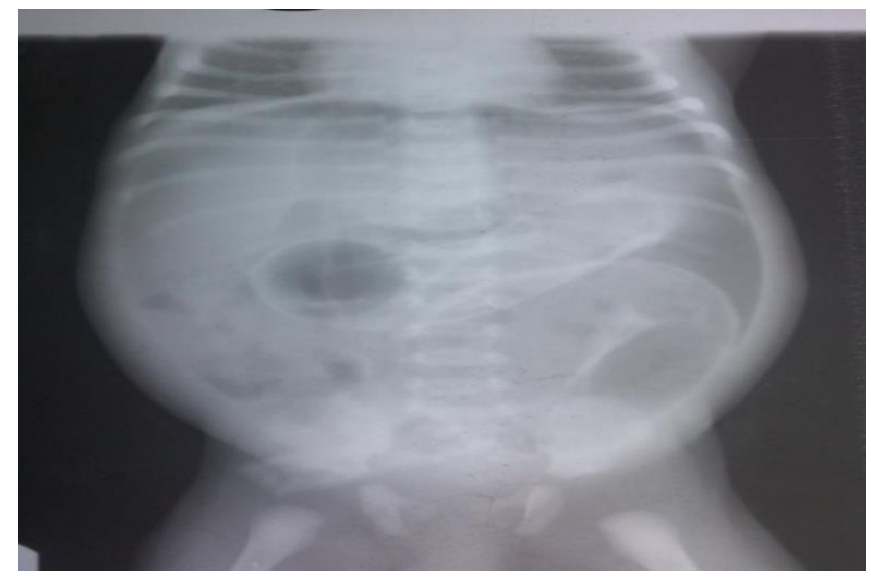

Figure 2 Supine abdominal radiograph showing Rigler's sign (curved arrow) and air trapped at the inferior margin of the liver (long arrow).

\section{Discussion}

Neonatal pneumoperitoneum has both surgical and non-surgical causes [4, 5]. In the newborns, it commonly arises from a perforated hollow viscus. It is also associated with pulmonary air leak syndromes- pneumothorax, pneumomediastinum, in neonates on mechanical ventilation. Pneumoperitoneum without viscus rupture is rare at any paediatric age [6]. Shalini et al reported a case of spontaneous idiopathic pneumoperitoneum in a newborn6. Reports have shown that necrotizing enterocolitis (NEC) is the single major cause of pneumoperitoneum in neonates [5, 7]. However, Khan in his study of 89 neonates managed for pneumoperitoneum showed that about $50 \%$ of patients had 
pneumoperitoneum due to non-NEC related cause. These other causes include perforated pouch colon, isolated colonic perforations, caecal perforation, gastric and duodenal perforations. However there were seven patients in which no cause could be ascertained [7]. No intra-abdominal cause of pneumoperitoneum was found in this index patient even during exploratory laparotomy. However the possibility of intrathoracic cause was not ruled out since chest radiograph was not done.

Plain abdominal radiograph is essential for the diagnosis of pneumoperitoneum. Erect abdomen or chest radiographs erect are required, but these are usually not obtained in neonates. The diagnosis in neonates is usually made with supine radiograph. Additional horizontal cross table shoot through films or left decubitus shoot through may be performed. Erect radiograph with the baby supported and a supine view were done in this patient to make the diagnosis.

The traditional sign of pneumoperitoneum is the crescent shaped free air beneath the diaphragm on erect abdominal and chest radiograph. In this position, it is possible to detect as little as 1 or $2 \mathrm{ml}$ of air [8]. This was demonstrated in the case here presented. The signs of pneumoperitoneum on supine abdominal radiograph include; the Dog's cap sign (air in Morrison's pouch), free air occurring sub-hepatically inferior and anterior to the liver, falciform ligament sign (air outlining the falciform ligament), wind sign or lucent liver sign, the dome sign (free air trapped under the middle part of the diaphragm), the double wall sign or Rigler's sign, the sign of triangle (air between the loops of bowel) and the football sign (air distended peritoneum beneath the anterior abdominal wall) [8, 9]. Some of these features (subhepatic air and Rigler's sign) were demonstrated in the case here presented.

Other conditions which can mimic the signs of pneumoperitoneum on plain radiograph are; interposition of the colon between the diaphragm and liver or Chilaiditi syndrome, fat depositions, artifacts, curvilinear pulmonary collapse, intraabdominal abscess, intraperitoneal or internal hernia and volvulus especially of the mobile caecum8.These can be differentiated from pneumoperitoneum by its described features.

The management of pneumoperitoneum depends on the cause. Laparotomy is done for pneumoperitoneum due to viscus perforation. NEC related perforations are managed by peritoneal drains. Conservative management (observation of vital signs, ceasation of enteric feeding and introduction of parenteral nutrition) can also be done in idiopathic cases. Shalini et al reported a case of spontaneous idiopathic pneumoperitoneum in the newborn which was managed conservatively [6]. However in the case presented the baby underwent exploratory laparotomy.

\section{Conclusion}

A 1 day old male neonate presented with gradual and progressive onset of abdominal swelling since birth and was diagnosed to have severe pneumoperitoneum by plain radiograph. He had exploratory laparotomy without any established cause identified.

\section{Compliance with ethical standards}

\section{Acknowledgments}

We acknowledge the assistance rendered by our health record staff in retrieving clinical case notes of these patients as well as departmental secretariat staff for their role in preparing the manuscript

\section{Disclosure of conflict of interest}

No conflict of interest

Statement of informed consent

Informed consent was obtained from the patients' parents

\section{References}

[1] Guillem P. Radiologic pneumoperitoneum without perforation of a hollow viscus. J Chir (Paris). 2002; 139 : 5-15.

[2] Ibrahim AI, Ahmed EA. Tapping for pneumoperitoneum in neonates and infants. Ann Paed Surg. 2013 ; 9: 47-53.

[3] Pati S, Chaki B, Roy A, De S. Can neonatal pneumoperitoneum be managed by conservative management alone? J Nep Paed Socie. 2012; 32: 252. 
[4] Mularski RA, Sippel JM, Osborne ML. Pneumoperitoneum: a review of non-surgical causes. Crit Care Med. 2000; 28: $2638-44$.

[5] Ekwunife OH, Ugwu J, Modekwe V, Osuigwe AN. Gastrointestinal perforation in neonates: Aetiology and risk factors. J Neonat Surg. 2013; 2: 30.

[6] Shalini A, Sunil T, Devend A. Spontaneous idiopathic pneumoperitoneum in a newborn. Paediatric oncall. 2012; 9: 4.

[7] Khan TR, Rawat JD, Ahmed IR, Kumar A, Maletha M, Wakhlu A, et al. Neonatal pneumoperitoneum: a critical appraisal of its causes and subsequent management from a developing country. Paed Surg Intern. 2009; $25: 1093$.

[8] Marija F, Tajana K, Ines M, Marijan F. Diagnostic value of pneumoperitoneum on plain abdominal film. Radiol Oncol. 2001; 35: 237-42.

[9] Shipee R, Suresh C, Teo Eu-leong H J, Victor S R. Radiological signs of pneumoperitoneum in an extremely low birthweight infant. Br Med J Case Reports. 2014. 